\title{
Preparation and Characterization of Lignin-containing Nanofibrillated Cellulose
}

\author{
Tianqing Lan, ${ }^{\mathrm{a}, \mathrm{b}}$ Haoran Liu, ${ }^{\mathrm{a}}$ Hui Li, ${ }^{\mathrm{a}}$ Yuyue Qin, ${ }^{\mathrm{a}}$ and Guojun Yue ${ }^{\mathrm{c}, *}$ \\ Lignin-containing nanofibrillated cellulose (LNFC) were prepared from $\mathrm{p}$ - \\ toluenesulfonic acid ( $\mathrm{p}-\mathrm{TsOH}$ ) pretreated sugarcane bagasse (SCB) using \\ either formic acid (FA) or hydrochloric acid $(\mathrm{HCl})$ and high-pressure \\ homogenization. The composition, morphology, dispersity, crystallinity, \\ particle size, thermal stability, and hydrophobicity of LNFC treated with FA \\ ( $\mathrm{F}$ - LNFC) and $\mathrm{HCl}(\mathrm{H}$ - LNFC) were compared via electron microscopy, an \\ X-ray diffractometer (XRD), a thermal gravimetric analyzer (TGA), a \\ Fourier transform infrared spectroscope (FTIR), and water contact angle \\ (WCA) analysis. The results of morphology and dispersity testing showed \\ that LNFC with uniform dispersion were successfully prepared using a \\ homogeneous pressure of $30 \mathrm{MPa}$ and the F- LNFC particles were more \\ stable in an aqueous solution. The crystallinity of the LNFC was well \\ maintained after homogenization. The TGA, FTIR, and WCA data \\ indicated that F-LNFC had better thermal stability and were more \\ hydrophobic than H-LNFC because FA could esterify cellulose. Improved \\ dispersity and thermal stability and increased crystallinity and \\ hydrophobicity of cellulose nanofibrils would enhance the performance of \\ nanocomposite materials.
}

Keywords: p-Toluenesulfonic acid pretreatment; Sugarcane bagasse; Formic acid; Hydrochloric acid; Lignin-containing cellulose nanofibrils; Cellulose nanofibril

Contact information: a: Faculty of Agriculture and Food, Kunming University of Science and Technology, 650500 Kunming, China; b: State Key Laboratory of Pulp and Paper Engineering, South China University of Technology, 510640 Guangzhou, China; c: SDIC Biotech Investment Co., Ltd., No. 147 Xizhimen

Nanxiao Street, Xicheng district, 100034 Beijing, China;

*Corresponding author: yueguojun_sdic@163.com

\section{INTRODUCTION}

Cellulose nanofibrils (NFC) have attracted attention due to their non-toxicity and biodegradability (Zhu et al. 2016). In most studies on NFC, the expensive bleached wood pulp is commonly used as the starting material (Bian et al. 2017a; Xie et al. 2018). Lignincontaining cellulose nanofibrils (LNFC) have better thermal stability and are more hydrophobic than NFC because they contain lignin that is more hydrophobic and more difficult to pyrolyze than cellulose and hemicellulose (Poletto et al. 2012; Rojo et al. 2015). Furthermore, non-bleached lignocellulose can be adopted as the raw material for the preparation of LNFC, which simplifies preparation and reduces cost (Rojo et al. 2015). Thus, LNFC should be thoroughly studied. Wood is the raw material typically used for LNFC preparation, but excessive logging damages the environment. Therefore, sugarcane bagasse, an industrial cellulose-containing by-product with abundant reserves, is a potential alternative raw material for the preparation of LNFC.

$\mathrm{p}$-Toluenesulfonic acid $(\mathrm{p}-\mathrm{TsOH})$ pretreatment is a novel lignocellulose treatment method that can remove $85 \%$ to $90 \%$ of lignin after 20 min of treatment at $80{ }^{\circ} \mathrm{C}$ (Bian et al. 2017a; Chen et al. 2017). This pretreatment causes little damage to cellulose, so the 
crystallinity and the proportion of cellulose are higher than most other treatment methods (Bian et al. 2017a). Furthermore, p-TsOH can be completely recycled as organic acid (Yang et al. 2019), which is environmentally friendly. Therefore, lignocellulose treated with $\mathrm{p}$ - $\mathrm{TsOH}$ can be regarded as a promising starting material for the preparation of cellulose nanofibrils.

This study investigated the feasibility of preparing LNFC using sugarcane bagasse (SCB) treated with p-TsOH as the starting material and applying formic acid (FA) or $\mathrm{HCl}$ treatment (the most common organic and inorganic acid treatment methods) and highpressure homogenization. The composition, morphology, crystallinity, dispersibility, particle size, chemical group, thermal stability, and hydrophobicity of the two LNFC were determined and analyzed. This study provides references for high-value utilization of SCB and preparation of high-performance LNFC.

\section{EXPERIMENTAL}

\section{Materials}

Sugarcane bagasse samples were collected from Yunnan Xinping Nanen Sugar Paper Co., Ltd. (Yunnan, China). Formic acid (FA) (88 wt\%), hydrochloric acid $(\mathrm{HCl})$ (37\%), and p-TsOH were purchased from Tianjin Wind Boat Chemical Reagent Technology Co., Ltd. (Tianjin, China). Carboxymethyl cellulose sodium (CMC) was purchased from Shanghai Aladdin Biochemical Technology Co., Ltd. (Shanghai, China). All chemicals were of analytical grade.

\section{Methods}

\section{Preparation of cellulose nanofibrils}

Sugarcane bagasse was ground and sieved with a 30-mesh screen and then pretreated with $65 \mathrm{wt} \% \mathrm{p}-\mathrm{TsOH}$ (ratio of 1:20) at $80{ }^{\circ} \mathrm{C}$ for $20 \mathrm{~min}$ (Bian et al. 2017a). The hydrolysis residue was washed to neutrality and marked as S-P. The S-P was treated with $88 \mathrm{wt} \%$ FA (ratio of 1:20) at $95^{\circ} \mathrm{C}$ for $6 \mathrm{~h}$ and then washed to neutrality (Du et al. 2016a). The solid residue was marked as F-LCSR. The S-P was treated with $2 \mathrm{M} \mathrm{HCl}$ at $80{ }^{\circ} \mathrm{C}$ for $2 \mathrm{~h}$, and the solid-liquid ratio of the S-P to $\mathrm{HCl}$ was 1:20 (w/v). Next, the solid residue was washed to neutrality and marked as H-LCSR. The F-LCSR and H-LCSR suspensions $(0.2$ wt $\%$ ) were homogenized 20 times with a high-pressure homogenizer (GJJ-0.06/70MPa; Shanghai Noni Light Industrial Machinery Co., Ltd., Shanghai, China) at $30 \mathrm{MPa}$. After homogenization, the F-LCSR and H-LCSR suspensions were referred to as F-LNFC and H-LNFC, respectively.

\section{Chemical composition}

The chemical compositions including the cellulose, hemicellulose, and lignin contents of S-P, F-LCSR/F-LNFC, and H-LCSR/H-LNFC were determined according to the method from the National Renewable Energy Laboratory (Sluiter et al. 2012).

Scanning electron microscope (SEM) and transmission electron microscope (TEM)

The microstructures of S-P, F-LCSR, and H-LCSR were imaged by SEM (NoVananoSEM450; FEI Company, Portland, OR, USA). The photos of the samples were taken at $5 \mathrm{kV}$. The microstructures of F-LNFC and H-LNFC were imaged by TEM (HT7700; Hitachi, Tokyo, Japan). The imaging of the samples was performed at $100 \mathrm{kV}$. 


\section{Particle size and zeta potential}

The particle size distribution and zeta potential of F-LNFC and H-LNFC suspensions were analyzed by a particle size and zeta potential analyzer (Zetasizer Nano ZS90; Malvern Instruments Limited, Malvern, England).

$X$-ray diffractometer (XRD)

The samples of S-P, F-LCSR, H-LCSR, F-LNFC, and H-LNFC were analyzed by $\mathrm{XRD}$ (D/Max 2200; Rigaku, Tokyo, Japan) with $\mathrm{Cu}-\mathrm{K} \alpha$ radiation $(\lambda=1.54 \AA$ ). The samples were scanned from $5^{\circ}$ to $90^{\circ}$ with a step size of $0.02^{\circ}$. The crystallinity index $(\mathrm{CrI})$ was calculated with Eq. 1,

$$
C r I=\left(\mathrm{I}_{002}-\mathrm{I}_{\mathrm{am}}\right) \times 100 \% / \mathrm{I}_{002}
$$

where $I_{002}$ is the scattered intensity of diffraction (002) plane and $I_{\mathrm{am}}$ is the minimum intensity between the main peak and the secondary peak.

Thermal gravimetric analyzer (TGA)

The thermal stabilities of S-P, F-LNFC, and H-LNFC were measured using a TGA (STA449 F3A00; Netzsch, Serb, Germany). Five mg of each sample was heated from ambient temperature to $600{ }^{\circ} \mathrm{C}$ in high purity nitrogen with a flow rate of $25 \mathrm{~mL} / \mathrm{min}$ at a heating rate of $10^{\circ} \mathrm{C} / \mathrm{min}$.

\section{Fourier transform infrared (FTIR) spectroscopy}

The chemical compositions of S-P, LCSR, and LNFC were evaluated by means of an FTIR spectroscope (TENSOR27; Bruker Karlsruhe, Baden-Wurttemberg, Germany) at $400 \mathrm{~cm}^{-1}$ to $4000 \mathrm{~cm}^{-1}$ with a resolution of $4 \mathrm{~cm}^{-1}$.

\section{Hydrophobicity}

The preparation of CMC film and the nanocomposite films (CMC/F-LNFC and CMC/H-LNFC) was as follows: $100 \mathrm{~mL}$ of LNFC suspension with a solid content of 0.1 wt $\%$ was mixed with $0.6 \mathrm{~g}$ of glycerol and $2 \mathrm{~g}$ of $\mathrm{CMC}$ for $4 \mathrm{~h}$ at room temperature. The mixture was poured onto a glass plate $(20 \mathrm{~cm} \times 20 \mathrm{~cm})$ and dried at $45^{\circ} \mathrm{C}$ overnight. Then, the films were kept at $25{ }^{\circ} \mathrm{C}$ and $50 \%$ relative humidity for $2 \mathrm{~d}$. The surface hydrophobicities of the films were measured using a contact angle meter (JY-82; Chengde Jinhe Instrument Manufacturing Co., Ltd., Hebei, China). The water contact angle (WCA) of the film was calculated as the average angle of at least five different positions.

\section{Statistical analysis}

All experiments and assays were performed in triplicate. Origin 8.5 software was used for data analysis (OriginLab, Northampton, MA, USA). Data were analyzed using one-way analysis of variance and Student's t-test. The alpha level was $p<0.05$.

\section{RESULTS AND DISCUSSION}

\section{Composition}

Lignocellulose treated with $\mathrm{p}-\mathrm{TsOH}$ often contains a high proportion of cellulose with a high crystallinity (Bian et al. 2017a). Therefore, it is a suitable raw material for the preparation of cellulose nanofibrils. In this study, F-LNFC and H-LNFC were prepared 
using p-TsOH-treated SCB. To investigate the characteristics of these two LNFC, the composition of lignocellulosic substrates was analyzed. In Table 1, the lignin, cellulose, and hemicellulose contents of S-P, F-LNFC, and H-LNFC are shown. It was found in Table 1 that, after FA and $\mathrm{HCl}$ treatment of S-P, the cellulose contents of F-LNFC and H-LNFC increased to $72.4 \%$ and $74.2 \%$ from $65.0 \%$, the hemicellulose contents decreased to $16.9 \%$ and $18.5 \%$ from $21.3 \%$, and the lignin contents were not significantly different $(p>0.05)$. The existence of lignin can make homogenization easier (Spence et al. 2010). In this study, the homogenization condition was $30 \mathrm{MPa}$, which is milder than most other studies (Du et al. 2016a).

Table 1. Composition of S-P, F-LCSR or F-LNFC, and H-LCSR or H-LNFC *

\begin{tabular}{|c|c|c|c|}
\hline Samples & Cellulose (\%) & Hemicellulose (\%) & Lignin (\%) \\
\hline S-P & $65.05 \pm 0.07^{\mathrm{a}}$ & $21.27 \pm 0.33^{\mathrm{a}}$ & $9.13 \pm 0.11^{\mathrm{a}}$ \\
\hline F-LCSR/F-LNFC & $72.43 \pm 0.62^{\mathrm{b}}$ & $16.87 \pm 0.24^{\mathrm{b}}$ & $9.19 \pm 0.17^{\mathrm{a}}$ \\
\hline H-LCSR/H-LNFC & $74.17 \pm 0.62^{\mathrm{c}}$ & $18.54 \pm 0.65^{\mathrm{c}}$ & $8.11 \pm 0.46^{\mathrm{a}}$ \\
\hline
\end{tabular}

${ }^{*}$ Data with the different superscripts denote a statistically significant difference $(p<0.05)$. The values following \pm are standard deviations. In this study, LNFC was produced using LCSR through homogenization. Therefore, the composition of LNFC was the same as that of LCSR.

\section{Morphology and Dispersity}

Figure 1a shows the microtopography of S-P, LCSR, and LNFC. The F-LCSR was smaller than the H-LCSR because formic acid degraded more cellulose and hemicellulose (Du et al. 2016a). The two LNFC exhibited a network structure, and H-LNFC had more agglomeration than F-LNRC. In addition, some lignin particles (black dots in the TEM photos of LNFC) were present (Bian et al. 2017b).

(a)

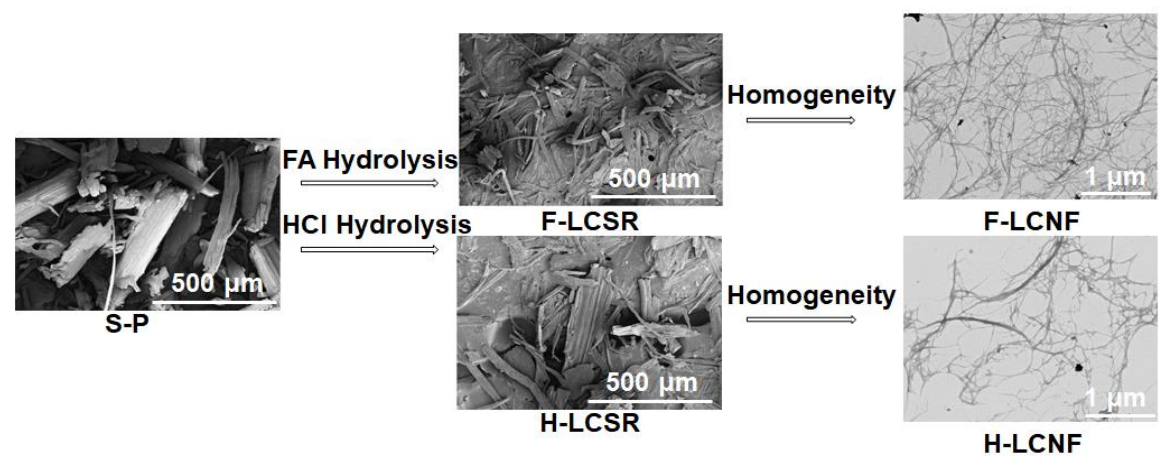

(b)
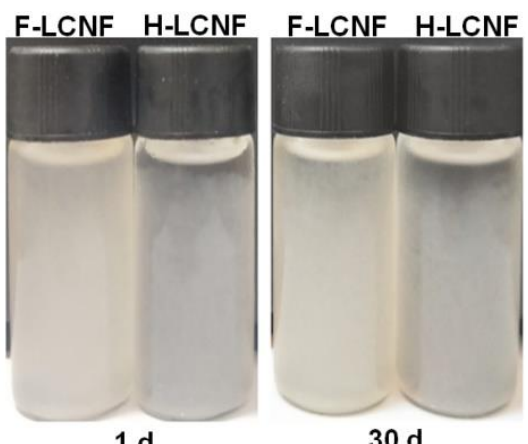

(c)

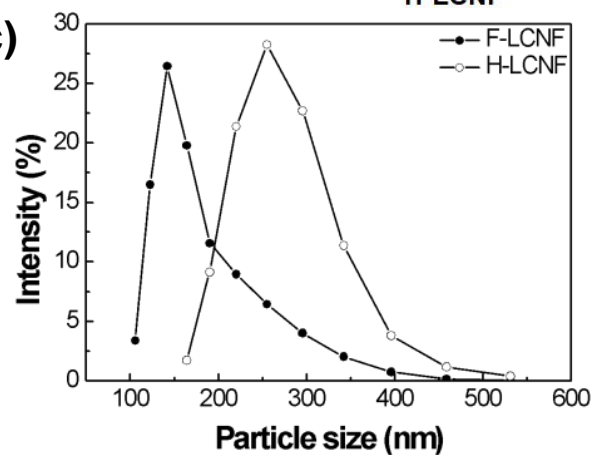

Fig. 1. SEM images of S-P and LCSR and TEM images of LNFC (a), LNFC suspensions after standing for $1 \mathrm{~d}$ and $30 \mathrm{~d}(\mathrm{~b})$, and particle size distributions of LNFC (c) 
Dispersity is an important property of LNFC. To analyze the effect of size and morphology on the dispersibility of LNFC, photos of LNFC suspensions were taken after $1 \mathrm{~d}$ and $30 \mathrm{~d}$ of standing (Fig. 1b), and the particle size distributions of LNFC were measured (Fig. 1c). The LNFC suspensions did not have notable sedimentation after standing for $1 \mathrm{~d}$ and $30 \mathrm{~d}$. The dispersity of F-LNFC was better than that of H-LNFC, which may have been due to its smaller size.

The dispersity of LNFC is influenced by surface charge and particle size. The average particle sizes and zeta potentials of LNFC are listed in Table 2. The zeta potential of F-LNFC was less negative than that of H-LNFC. The lower absolute value of zeta potential implies less electrostatic repulsion between the nanoparticles, which had a negative effect on the dispersion of LNFC particles (Liu et al. 2014). However, the small average size of F-LNFC $(174.23 \mathrm{~nm})$ relative to that of the H-LNFC $(267.71 \mathrm{~nm})$, was beneficial to the dispersion of nanofibrils (Jia et al. 2017). In other studies, the average particle sizes of cellulose nanocrystals ranged from $150 \mathrm{~nm}$ to $375 \mathrm{~nm}$ (Du et al. 2016b; Oun and Rhim 2017). In this study, F-LNFC had a relatively small average size and better dispersity. The smaller size could impact the uniform distribution of LNFC in suspension and composite materials (Oun and Rhim 2017).

Table 2. Average Particle Sizes of F-LNFC and H-LNFC*

\begin{tabular}{|c|c|c|}
\hline Samples & F-LNFC & H-LNFC \\
\hline Zeta Potential $(\mathrm{mV})$ & $-9.96 \pm 1.75^{\mathrm{a}}$ & $-29.63 \pm 4.02^{\mathrm{b}}$ \\
\hline Average Particle Size $(\mathrm{nm})$ & $174.23 \pm 12.15^{\mathrm{a}}$ & $267.71 \pm 8.47^{\mathrm{b}}$ \\
\hline
\end{tabular}

* Data with the different superscripts denote a statistically significant difference $(p<0.05)$. The values following \pm are standard deviations.

\section{Crystallinity}

Because the crystallinity of cellulose nanofibrils can influence the properties of nanocellulose materials, the crystallinities of S-P, LCSR, and LNFC were measured. Figure 2 shows that after $\mathrm{S}-\mathrm{P}$ was treated with $\mathrm{FA}$ and $\mathrm{HCl}$, the crystallinities of F-LCSR and $\mathrm{H}-$ LCSR were $68.7 \%$ and $71.4 \%$, respectively, which were higher than the $62.67 \%$ crystallinity of S-P. This was because FA and $\mathrm{HCl}$ removed some non-crystalline parts of the S-P substrate.

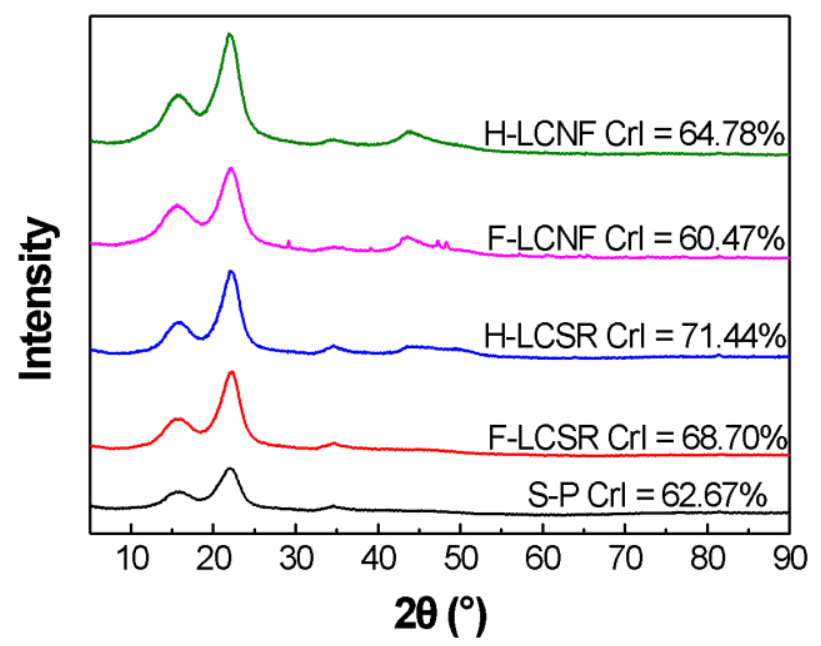

Fig. 2. The XRD spectrums of S-P, LCSR, and LNFC 
After homogenization, the crystallinities of F-LNFC and H-LNFC decreased to $60.5 \%$ and $64.8 \%$, respectively, because high pressure homogenization can destroy the highly ordered crystalline region structure of the fiber by strong shear force (Saelee et al. 2016) and reduce the crystallinity (Hoeger et al. 2013).

After mild homogenization at $30 \mathrm{MPa}$, the crystallinities of F-LNFC and H-LNFCF decreased from $68.7 \%$ and $71.4 \%$ to $60.5 \%$ and $64.8 \%$, respectively. This occurred because homogenization damaged the crystallization zone of cellulose (Saelee et al. 2016). However, the decrease in crystallinity observed in this study was lower than that of other studies due to using a relatively low homogenization pressure. The crystallinities of cellulose nanofibrils in previous studies ranged from 49.9\% to 57.1\% (Du et al. 2016a; Liu et al. 2016). Higher crystallinities enhance the mechanical properties of nanocomposites (Talbott et al. 1987).

\section{Thermal Stability}

To analyze the thermal stability of F-LNFC and H-LNFC, the initial pyrolysis temperature $\left(T_{\text {onest }}\right)$ and maximum pyrolysis temperature $\left(T_{\max }\right)$ of S-P and LNFC were measured. The thermogravimetric (TG) and derivative thermogravimetric (DTG) curves of the samples are shown in Fig. 3. The $T_{\text {onest }}$ and $T_{\max }$ of the samples are listed in Table 3. Figure 3 shows that there was slight weight loss at $100{ }^{\circ} \mathrm{C}$. This was due to evaporation and the removal of water absorbed by the sample (Chirayil et al. 2014). Table 3 indicates that the initial and maximum pyrolysis temperatures of F-LNFC and H-LNFC were higher than those of S-P. Generally, the crystalline cellulose with high crystallinity had better thermal stability (Du et al. 2016b). In this study F-LNFC had lower crystallinity compared with H-LNFC and S-P; however, the thermal stability of F-LNFC was better than H-LNFC and S-P. This was because the thermal stability of cellulose nanoparticles was affected by not only crystallinity but also esterification of nanoparticles (Du et al. 2016b; Sun et al. 2016). Therefore, the better thermal stability of F-LNFC might be because that FA esterified S-P and made F-LNFC possess ester groups. It was reported in the literature (Sun et al. 2008), when the ester groups with better thermal stability replaced the hydroxyl groups with poor thermal stability on cellulose, the thermal stability of cellulose would be improved.
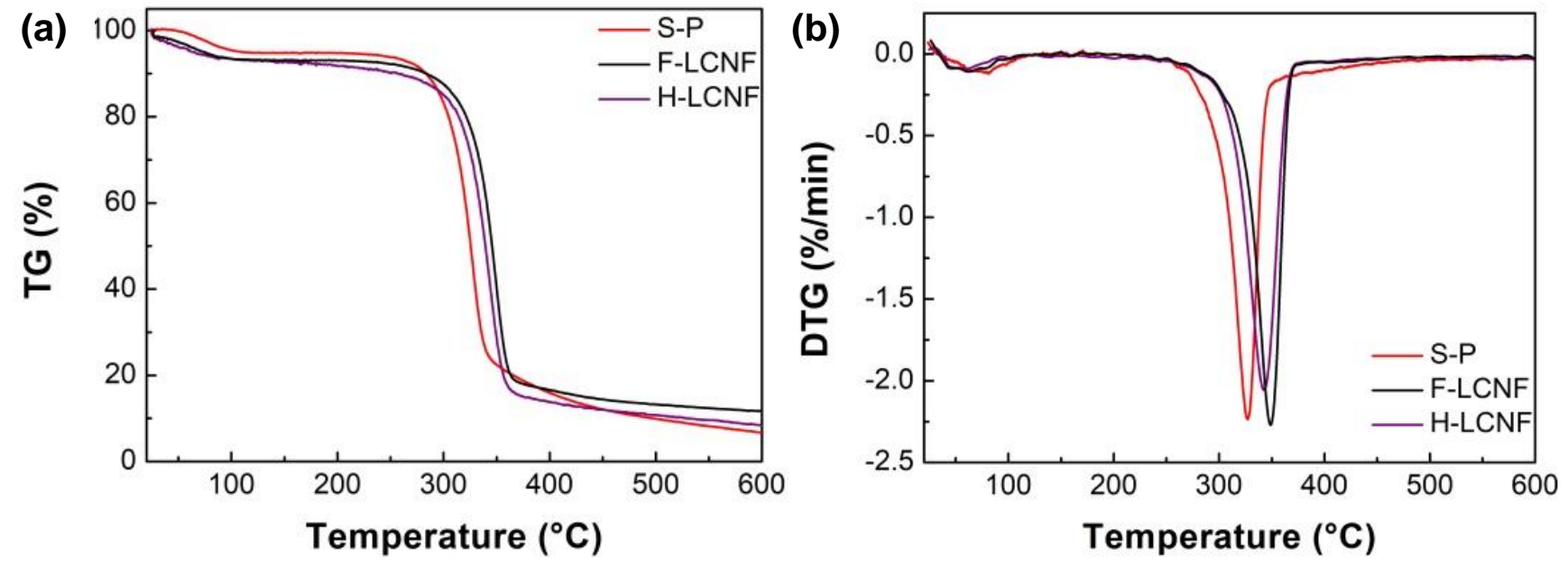

Fig. 3. The (a) TG and (b) DTG curves of S-P, F-LNFC, and H-LNFC 
The F-LNFC and H-LNFC in this study had higher thermal stability compared with the reported research. Jonoobim et al. (2015) summarized the thermal stability of more than a dozen NFC prepared with different raw materials and methods and found that the initial pyrolysis temperature and the maximum pyrolysis temperature ranged from $209{ }^{\circ} \mathrm{C}$ to $335{ }^{\circ} \mathrm{C}$ and $300{ }^{\circ} \mathrm{C}$ to $351{ }^{\circ} \mathrm{C}$, respectively. The initial pyrolysis temperature and maximum pyrolysis temperature of F-LNFC were $309.09^{\circ} \mathrm{C}$ and $350.00{ }^{\circ} \mathrm{C}$, respectively, and these of $\mathrm{H}$-LNFC were $300.00{ }^{\circ} \mathrm{C}$ and $341.67{ }^{\circ} \mathrm{C}$, respectively. The phenyl in lignin was difficult to pyrolyze, which improved the thermal stability of LNFC (Poletto et al. 2012; Deepa et al. 2015). High thermal stability enhanced the thermoplasticity of the materials (Brinchi et al. 2013).

Table 3. Thermal Stabilities of the S-P, F-LNFC, and H-LNFC

\begin{tabular}{|c|c|c|}
\hline Samples & $T_{\text {onset }}\left({ }^{\circ} \mathrm{C}\right)$ & $T_{\max }\left({ }^{\circ} \mathrm{C}\right)$ \\
\hline S-P & 286.36 & 327.08 \\
\hline F-LNFC & 309.09 & 350.00 \\
\hline H-LNFC & 300.00 & 341.67 \\
\hline
\end{tabular}

\section{FTIR Analysis}

The FTIR spectra of S-P and LNFC were determined (Fig. 4) to analyze the effect of $\mathrm{FA}$ and $\mathrm{HCl}$ treatment on the functional groups. Figure 4 shows that the F-LNFC spectrum showed a peak at $1720 \mathrm{~cm}^{-1}$ that represents the extension of ester group $\mathrm{C}=\mathrm{O}$ in cellulose treated by FA (Sun et al. 2008). This was because when FA hydrolyzed S-P, the hydroxyl groups in the carboxyl groups of FA were combined with the hydrogen atoms in the hydroxyl groups of the cellulose and ester groups were formed (Sun et al. 2008).

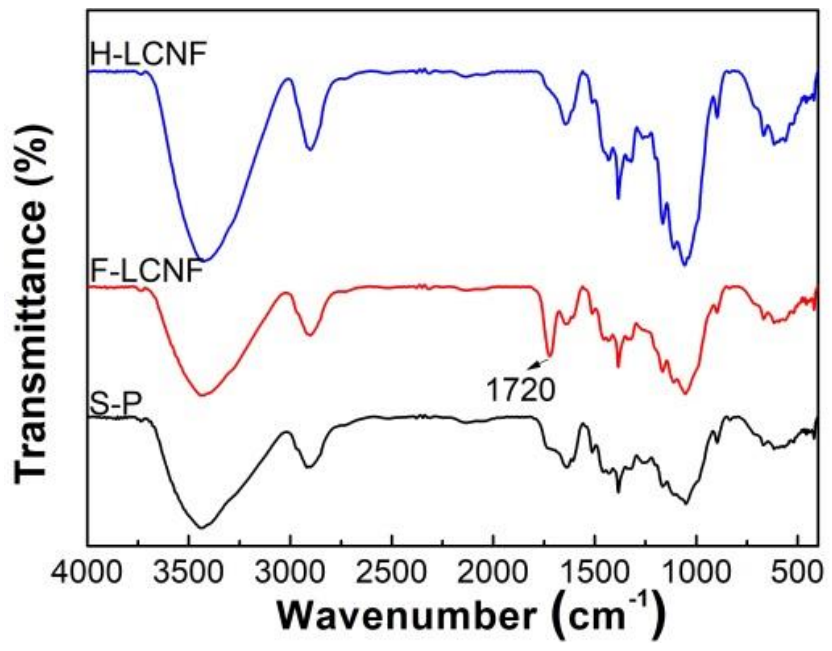

Fig. 4. The FTIR spectra of S-P, F-LNFC, and H-LNFC

\section{Hydrophobicity}

To analyze the hydrophobic properties of F-LNFC and H-LNFC, the WCA of CMC, F-LNFCF/CMC, and H-LNFC/CMC were measured using CMC as the blank carrier. The WCAs are listed in Table 4. The WCA of blank CMC film was $62.7^{\circ}$, which indicates that it was hydrophilic. After adding F-LNFC and H-LNFC, the WCAs of films increased to $102.7^{\circ}$ and $111.2^{\circ}$, respectively $(\mathrm{p}<0.05)$, which indicates that the films were more hydrophobic after adding LNFC. Lignin can affect the hydrophobicity of cellulose, 
and LNFC is generally more hydrophobic than NFC (Spence et al. 2010; Poletto et al. 2012). Table 4 also shows that the WCA of F-LNFC/CMC was larger than that of $\mathrm{H}$ $\mathrm{LFC} / \mathrm{CMC}(\mathrm{p}<0.05)$. The lignin contents of these two LNFC had no significant difference. This may have been due to F-LNFC containing more ester groups, which are hydrophobic (Scarica et al. 2018).

\section{Table 4. Water Contact Angle of LNFC Films *}

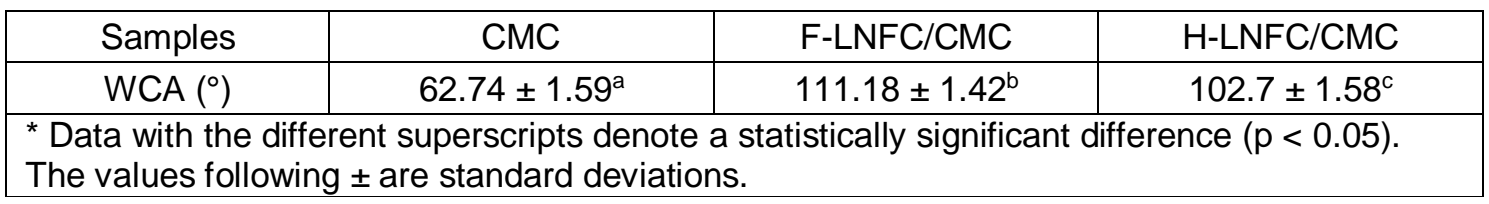

\section{CONCLUSIONS}

1. The formic acid-treated lignin-containing nanofibrillated cellulose (F-LNFC) prepared from $\mathrm{p}$ - $\mathrm{TsOH}$ pretreated sugarcane bagasse (S-P) had better dispersity than the HClpretreated version (H-LNFC), which was attributed to the smaller particle size of the F-LNFC.

2. The LNFC in this study had higher crystallinity and better thermal stability than those reported in other studies.

3. The F-LNFC was more hydrophobic than the H-LNFC because formic acid esterified cellulose and formed more ester groups in the F-LNFC.

\section{ACKNOWLEDGMENTS}

This work was supported by the National Natural Science Foundation of China (Grant No. 21766014) and the State Key Laboratory of Pulp and Paper Engineering (Grant No. 201811).

\section{REFERENCES CITED}

Bian, H. Y., Chen, L. H., Gleisner, R., Dai, H. Q., and Zhu, J. Y. (2017a). "Producing wood-based nanomaterials by rapid fractionation of wood at $80^{\circ} \mathrm{C}$ using a recyclable acid hydrotrope," Green Chemistry 19(14), 3370-3379. DOI: 10.1039/c7gc00669a

Bian, H. Y., Chen, L. H., Dai, H. Q., and Zhu, J. Y. (2017b). "Integrated production of lignin containing cellulose nanocrystals (LCNC) and nanofibrils (LCNF) using an easily recyclable di-carboxylic acid," Carbohydrate Polymers 167, 167-176. DOI: 10.1016/j.carbpol.2017.03.050

Brinchi, L., Cotana, F., Fortunati, E., and Kenny, J. M. (2013). "Production of nanocrystalline cellulose from lignocellulosic biomass: Technology and applications," Carbohydrate Polymers 94(1), 154-169. DOI:

10.1016/j.carbpol.2013.01.033

Chen, L. H., Dou, J. H., Ma, Q. L., Li, N., Wu, R. C., Bian, H. Y., Yelle, D. J., Vuorinen, T., Fu, S. Y., Pan, X. J., et al. (2017). "Rapid and near-complete dissolution of wood 
lignin at $\leq 80{ }^{\circ} \mathrm{C}$ by a recyclable acid hydrotrope," Science Advances 3(9), 1-11. DOI: 10.1126/sciadv.1701735

Chirayil, C. J., Joy, J., Mathew, L., Mozetic, M., Koetz, J., and Thomas, S. (2014). "Isolation and characterization of cellulose nanofibrils from Helicteres isora plant," Industrial Crops and Products 59, 27-34. DOI: 10.1016/j.indcrop.2014.04.020

Deepa, B., Abraham, E., Cordeiro, N., Mozetic, M., Mathew, A. P., Oksman, K., Faria, M., Thomas, S., and Pothan, L. A. (2015). "Utilization of various lignocellulosic biomass for the production of nanocellulose: A comparative study," Cellulose 22(2), 1075-1090. DOI: 10.1007/s10570-015-0554-X

Du, H. S., Liu, C., Zhang, Y. D., Yu, G., Si, C. L., and Li, B. (2016a). "Preparation and characterization of functional cellulose nanofibrils via formic acid hydrolysis pretreatment and the followed high-pressure homogenization," Industrial Crops and Products 94, 736-745. DOI: 10.1016/j.indcrop.2016.09.059

Du, H. S., Liu, C., Mu, X. D., Gong, W. B., Lv, D., Hong, Y. M., Si, C. L., and Li, B. (2016b). "Preparation and characterization of thermally stable cellulose nanocrystals via a sustainable approach of $\mathrm{FeCl}_{3}$-catalyzed formic acid hydrolysis," Cellulose 23(4), 2389-2407. DOI: 10.1007/s10570-016-0963-5

Hoeger, I. C., Nair, S. S., Ragauskas, A. J., Deng, Y. L., Rojas, O. J., and Zhu, J. Y. (2013). "Mechanical deconstruction of lignocellulose cell walls and their enzymatic saccharification," Cellulose 20(2), 807-818. DOI: 10.1007/s10570-013-9867-9

Jia, C., Chen, L. H., Shao, Z. Q., Agarwal, U. P., Hu, L. B., and Zhu, J. Y. (2017). "Using a fully recyclable dicarboxylic acid for producing dispersible and thermally stable cellulose nanomaterials from different cellulosic sources," Cellulose 24(6), 24832498. DOI: 10.1007/s10570-017-1277-y

Jonoobim, M., Oladi, R., Davoudpour, Y., Oksman, K., Durfresne, A., Hamzeh, Y., and Davoodi, R. (2015). "Different preparation methods and properties of nanostructured cellulose from various natural resources and residues: A review," Cellulose 22(2), 935-969. DOI: 10.1007/s10570-015-0551-0

Liu, Y. F., Wang, H. S., Yu, G., Yu, Q. X., Li, B., and Mu, X. D. (2014). “A novel approach for the preparation of nanocrystalline cellulose by using phosphotungstic acid," Carbohydrate Polymers 110, 415-422. DOI: 10.1016/j.carbpol.2014.04.040

Liu, C., Li, B., Du, H. S., Lv, D., Zhang, Y. D., Yu, G., Mu, X. D., and Peng, H. (2016). "Properties of nanocellulose isolated from corncob residue using sulfuric acid, formic acid, oxidative and mechanical methods," Carbohydrate Polymers 151, 716-724. DOI: 10.1016/j.carbpol.2016.06.025

Oun, A. A., and Rhim J.-W. (2017). "Characterization of carboxymethyl cellulose-based nanocomposite films reinforced with oxidized nanocellulose isolated using ammonium persulfate method," Carbohydrate Polymers 174, 484-492. DOI: 10.1016/j.carbpol.2017.06.121

Poletto, M., Zattera, A. J., Forte, M. M. C., and Santana, R. M. C. (2012). "Thermal decomposition of wood: Influence of wood components and cellulose crystallite size," Bioresource Technology 109, 148-153. DOI: 10.1016/j.biortech.2011.11.122

Rojo, E., Peresin, M. S., Sampson, W. W., Hoeger, I. C., Vartiainen, J., Laine, J., and Rojas, O. J. (2015). "Comprehensive elucidation of the effect of residual lignin on the physical, barrier, mechanical and surface properties of nanocellulose films," Green Chemistry 17(3), 1853-1866. DOI: 10.1039/c4gc02398f

Saelee, K., Yingkamhaeng, N., Nimchua, T., and Sukyai, P. (2016). “An environmentally friendly xylanase-assisted pretreatment for cellulose nanofibrils isolation from 
sugarcane bagasse by high-pressure homogenization," Industrial Crops and Products 82, 149-160. DOI: 10.1016/j.indcrop.2015.11.064

Scarica, C., Suriano, R., Levi, M., Turri, S., and Griffini, G. (2018). 'Lignin functionalized with succinic anhydride as building block for biobased thermosetting polyester coatings," ACS Sustainable Chemistry \& Engineering 6(3), 3392-3401. DOI: 10.1021/acssuschemeng.7b03583

Sluiter, A., Hames, B., Ruiz, R., Scarlata, C., Sluiter, J., Templeton, D., and Crocker, D. (2012). Determination of Structural Carbohydrates and Lignin in Biomass (NREL/TP-510-42618), National Renewable Energy Laboratory, Golden, CO, USA.

Spence, K. L., Venditti, R. A., Rojas, O. J., Habibi, Y., and Pawlak, J. J. (2010). "The effect of chemical composition on microfibrillar cellulose films from wood pulps: Water interactions and physical properties for packaging applications," Cellulose 17(4), 835-848. DOI: 10.1007/s10570-010-9424-8

Sun, B., Yu, H. Y., Zhou, Y., Huang, Z., and Yao, J. M. (2016). "Single-step extraction of functionalized cellulose nanocrystal and polyvinyl chloride from industrial wallpaper wastes," Industrial Crops and Products 89, 66-77. DOI: 10.1016/j.indcrop.2016.04.040

Sun, Y., Lin, L., Deng, H. B., Li, J. Z., He, B. H., Sun, R. C., and Ouyang, P. K. (2008). "Structural changes of bamboo cellulose in formic acid," BioResources 3(2), 297 315. DOI: 10.15376/biores.3.2.297-315

Talbott, M. F., Springer, G. S., and Berglund, L. A. (1987). "The effects of crystallinity on the mechanical properties of PEEK polymer and graphite fiber reinforced PEEK," Journal of Composite Materials 21(11), 1056-1081. DOI: 10.1177/002199838702101104

Xie, H. X., Du, H. S., Yang, X. G., and Si, C. L. (2018). "Recent strategies in preparation of cellulose nanocrystals and cellulose nanofibrils derived from raw cellulose materials," International Journal of Polymer Science 2018, 1-25. DOI: $10.1155 / 2018 / 7923068$

Yang, M. Y., Gao, X. F., Lan, M., Dou, Y., and Zhang, X. (2019). "Rapid fractionation of lignocellulosic biomass by p-TsOH pretreatment," Energy \& Fuels 33(3), 22582264. DOI: $10.1021 /$ acs.energyfuels.8b03770

Zhu, H., Luo, W., Ciesielski, P. N., Fang, Z., Zhu, J. Y., Henriksson, G., Himmer, M. E., and $\mathrm{Hu}$, L. (2016). "Wood-derived materials for green electronics, biological devices, and energy applications," Chemical Reviews 116(16), 9305-9374. DOI: 10.1021/acs.chemrev.6b00225

Article submitted: February 17, 2020; Peer review completed: April 11, 2020; Revised version received and accepted: April 29, 2020; Published: May 5, 2020.

DOI: 10.15376/biores.15.3.4689-4698 